\title{
Tratamiento laparoscópico de tres pacientes con tumores neuroendócrinos gástricos. Reporte de casos.
}

Laparoscopic treatment of three patients with gastric neuroendocrine tumors. Case report.

\author{
Martín Varela Vega ${ }^{1}$ \\ ORCID https://orcid.org/0000-0003-2376-2844 \\ Leticia Barro ${ }^{2}$ \\ ORCID https://orcid.org/0000-0001-6940-0342 \\ Sofía Baranov ${ }^{3}$ \\ ORCID https://orcid.org/0000-0002-1914-3285 \\ Agustina Da Rosa ${ }^{4}$ \\ ORCID https://orcid.org/0000-0002-7313-1306 \\ Gabriela Larrosa ${ }^{5}$ \\ ORCID https://orcid.org/0000-0003-1725-5096 \\ Santiago Cubas ${ }^{6}$ \\ ORCID https://orcid.org/0000-0001-6026-5316 \\ Gerardo Beraldo ${ }^{7}$ \\ ORCID https://orcid.org/0000-0002-3311-160X \\ Pablo Santiago ${ }^{8}$ \\ ORCID https://orcid.org/0000-0002-7733-8291
}

DOI: $10.31837 /$ cir.urug/5.1.6

\section{Resumen}

Los tumores neuroendócrinos gástricos constituyen el 6,9\% de todos los tumores neuroendócrinos del tubo digestivo, y el $1 \%$ de todas las neoplasias gástricas aproximadamente. Comprenden un amplio grupo de tumores que pueden ser secretantes o no, y con muy distinto comportamiento biológico. Si bien la mayoría de ellos son benignos, un pequeño porcentaje tiene un comportamiento agresivo, con capacidad metastásica y una pobre sobrevida. Dado su curso relativamente indolente, en ocasiones el diagnóstico resulta de un hallazgo fortuito, o menos comúnmente cuando surge alguna complicación (hemorragia u obstrucción). Para el diagnóstico es imprescindible la endoscopía alta con el análisis inmunohistoquímico de la biopsia. La tomografía es de elección para la estadificación. La diseminación ganglionar es infrecuente, y no está indicado un vaciamiento linfático a no ser en casos de tumores indiferenciados o con adenopatías visibles en el intraoperatorio, por lo que el abordaje laparoscópico resulta especialmente atractivo. El objetivo de esta comunicación es presentar tres casos de tumores neuroendócrinos de localización gástrica de resolución laparoscópica en nuestro servicio.

Palabras clave: tumores gástricos, cirugía gástrica, tratamiento quirúrgico

${ }_{1,2,3,4,5,6,7,8}$ Seccional de Cirugía Esófago Gástrica y Bariátrica. Hospital Central de las Fuerzas Armadas. Montevideo, Uruguay. martinvarelav@gmail.com 


\begin{abstract}
Gastric neuroendocrine tumors constitute $6.9 \%$ of all gastrointestinal neuroendocrine tumors and approximately $1 \%$ of all gastric neoplasms. They comprise a wide group of tumors that may or may not be secreting, and with very different biological behavior. Although most of them are benign, a small percentage have an aggressive behavior, with metastatic capacity and a poor survival. Given its relatively indolent course, sometimes the diagnosis results from a fortuitous finding, or less commonly when a complication (hemorrhage or obstruction) arises. Upper endoscopy with immunohistochemical analysis of the biopsy is essential for diagnosis. Tomography is the choice for staging. Lymph node dissemination is rare, and lymphatic emptying is not indicated except in cases of undifferentiated tumors or with visible lymphadenopathy intraoperatively, so the laparoscopic approach is especially attractive. The objective of this communication is to present three cases of laparoscopic resolution of gastric neuroendocrine tumors in our department.
\end{abstract}

Key words: gastric tumors, gastric surgery, surgical treatment

\title{
Introducción
}

El estómago es sitio de localización frecuente de tumores, tanto benignos como de diversa gama de malignidad. La estirpe más frecuente es el adenocarcinoma, en torno al 90-95\% de todos los tumores malignos gástricos. En menor proporción asientan en él otros tipos tumorales, tanto epiteliales como no epiteliales, que por su escasa frecuencia y heterogeneidad, constituyen un verdadero desafío diagnóstico y terapéutico. Los tumores neuroendócrinos gástricos (TNE) representan el $1 \%$ de todas las neoplasias gástricas aproximadamente $(1,2)$.

Son tumores de estirpe epitelial y pueden encontrarse a lo largo de todo el tubo digestivo, siendo el estómago uno de los sitios de localización más comunes $(6,9 \%)(3)$. En el estómago pueden originarse tanto de las células Entero Cromafín Like (ECL) o en las células G, productoras de gastrina. Es conocido el efecto trófico de la gastrina sobre las células ECL, por lo que los estados de hipergastrinemia resultantes de la respuesta de la células $G$ antrales a la aclorhidia, o a la hipersecreción de los gastrinomas, generan hiperplasia de estas células, y tienen relación con algunos tipos de TNE (1 y 2 específicamente).

En general estos tumores tienen un curso clínico silente debido a que la mayoría no son secretantes, siendo su diagnóstico un hallazgo en una endoscopía por otra causa. Otras veces se manifiestan por anemia o sangrado digestivo. El síndrome carcinoide (rash cutáneo, diarreas, taquicardia) se ve en algunos casos de TNE con hipersecreción de serotonina (8-28\%), y es un elemento de mal pronóstico ya que habitualmente indica secundarismo hepático (4).

Hasta hace algunos años reinó cierta confusión en la nomenclatura de estos tumores, antiguamente llamados APUDomas, o tumores carcinoides. En el año 2010 la OMSlos clasifica como tumores neuroendócrinosgastroenteropancreáticos (TNE GEP), reservando el término carcinoide para los de buen pronóstico (grado 1 y 2). Tanto la OMS como la ENETS (Asociación Europea de Tumores Neuroendócrinos) aplican para la comprensión de estos tumores una clasificación en base a la diferenciación celular, el grado de mitosis por campo de gran aumento y el índice inmunohistoquímico Ki67 (5). 
De esa manera se diferencian tres tipos de TNEGEP de distinto grado y comportamiento biológico.

a) Los TNE bien diferenciados GRADO 1, con atipía celular leve, bajo índice mitótico (1-2 mitosis/10 CGA) y un bajo índice proliferativo indicado por un ki67 <2\%.

b) Los TNE moderadamente diferenciados GRADO 2, con atipía celular evidente y actividad mitótica más importante (2-20 mitosis/10 CGA) y un ki67 entre 3 a 20\%.

c) Los TNE poco diferenciados GRADO 3 (o carcinoma neuroendócrino) con marcada atipía, abundantes mitosis (>20 mitosis/10 CGA) y un índice proliferativo ki67 > 20\%.

Dentro de los TNE gástricos es clásico realizar una segunda clasificación, tomando en cuenta la forma de presentación endoscópica y su relación con la hipergastrinemia (1)

a) Tipo 1: asociados a gastritis atrófica crónica (75\% de todos los TNE, usualmente benignos, múltiples y menores a $1 \mathrm{~cm}$ de tamaño).

b) Tipo 2: asociados a síndrome de múltiples neoplasias endócrinas (MEN-1) o al síndrome de Zollinger-Ellison (6\% de todos los TNE gástricos, pueden ser múltiples y generalmente son menores a $1,5 \mathrm{~cm})$.

c) Tipo 3: esporádicos, no asociados a hipergastrinemia ni a gastritis atrófica. Pueden presentar un curso maligno con infiltración de los planos profundos (76\% invaden la capa muscular) y diseminación ganglionar o metastásica. En el caso de ser productores de aminas biológicas pueden ocasionar un síndrome carcinoide.

El número de mitosis por 10 campos de gran aumento y el índice de proliferación Ki 67 indican el potencial agresivo de estos tumores, separando los de bajo potencial (grado 1 y 2, llamados también carcinoides), de los de alto potencial maligno (grado 3 o carcinoma neuroendócrino, que tienen más de 10 mitosis por cada 10CGA y un Ki 67 mayor a 20).(6)

A la histología, los tumores tipo 1 y 2 tienen un patrón celular regular, usualmente en mosaico, con núcleos monomórficos "en sal y pimienta", escasas mitosis y sin angioinvasión. No suelen pasar de la capa submucosa. En la inmunohistoquímica (IHQ) suelen tener positividad intensa y difusa para ckcocktail por su estirpe epitelial, y también para cromogranina A (CgA) y sinaptofisina. Los carcinomas neuroendócrinos en cambio, tienen marcada actividad mitótica, atipía celular y nuclear, invasión angiolinfática y ocasionalmente infiltración de plano muscular o seroso (7).

\section{Casos clínicos}

Presentamos a continuación tres pacientes con TNE gástricos diagnosticados y tratados en el servicio de cirugía esófago gástrica del HCFFAA.

\section{Paciente 1}

Se trataba de un hombre de 66 años, que consulta en la emergencia por cuadro de dolor abdominal en la fosa ilíaca derecha. Se realiza una TC de abdomen y pelvis que evidencia una apendicitis aguda, 
litiasis vesicular sin elementos de complicación y como hallazgo un engrosamiento antral de $3 \mathrm{~cm}$ aproximadamente, heterogéneo, que capta contraste iv (Fig 1). Se procede a la apendicectomía laparoscópica de urgencia, con buena evolución y alta a las 48 hs. En el postoperatorio se realiza endoscopía alta que visualiza la lesión de apariencia submucosa, de $3 \mathrm{~cm}$ aproximadamente, sobre la curvatura menor, en el contexto de una gastritis erosiva.
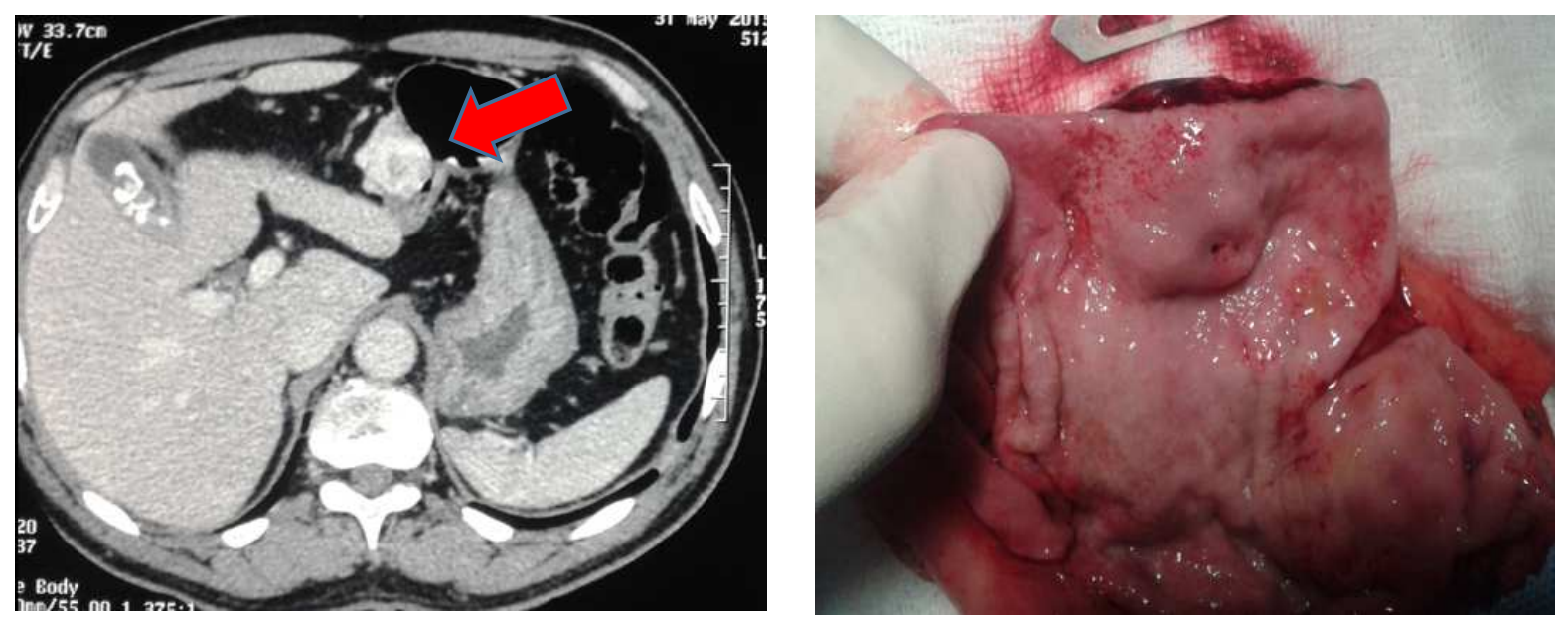

Fig. 1: TC abdómino pélvica con contrasteiv: hallazgo tumoración heterogénea en antro gástrico, sin adenopatías regionales, que capta contraste en fase venosa.

Fig. 2: Pieza de AP: tumor antral de $3 \mathrm{~cm}$ con umbilicación central, sin ulceración.

El informe de anatomía patológica de la biopsia endoscópica muestra mucosa gástrica normal. Ante el hallazgo sospechoso de un tumor submucoso gástrico se decide proceder con una cirugía resectiva. Se realiza una gastrectomía distal laparoscópica, con anastomosis gastroyeyunal en Y de Roux.

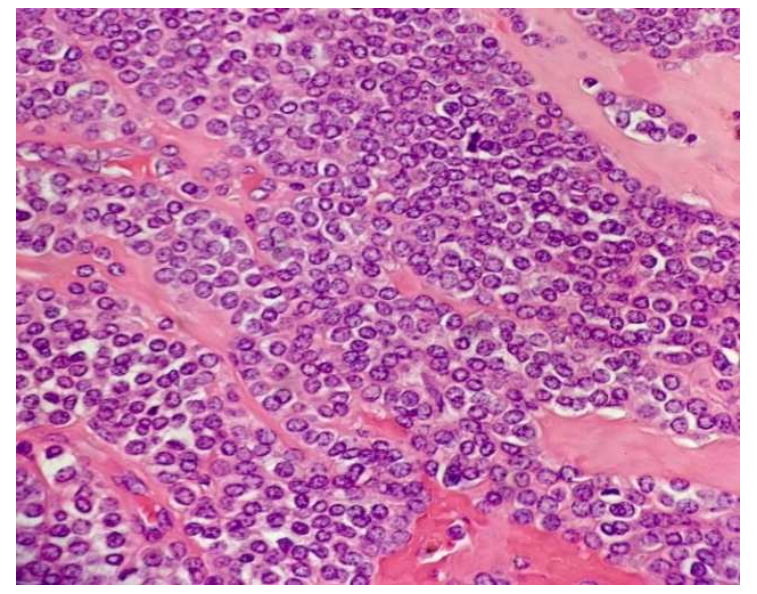

La anatomía patológica definitiva informa un TNE gástrico de $29 \mathrm{~mm}$ que infiltra la serosa (pT4N0) grado 1 de la OMS, con 2 mitosis cada 10 CGA y un Ki 67 menor al $1 \%$. En la IHQ muestra positividad intensa para $\mathrm{CgA}$ y positividad moderada para sinaptofisina.

monomórficos en sal y pimienta (patrón dotlike) característicos de un TNE bien diferenciado.

\section{Paciente 2}

Mujer de 54 años con antecedente de litiasis vesicular sintomática, que consulta por una hemorragia digestiva alta con repercusión hemodinámica y que requiere transfusión sanguínea. La endoscopía muestra una pequeña lesión de $1 \mathrm{~cm}$ ulcerada y de bordes sobreelevados en la curvatura mayor gástrica, con estigmas de sangrado reciente. La ecoendoscopía informa infiltración de la capa 
muscular (uT2N0), y la biopsia un TNE gástrico bien diferenciado grado 1. Se solicita TC de abdomen y pelvis que no evidencia diseminación ganglionar ni sistémica.

Se realiza una gastrectomía atípica de la curvatura mayor con endoscopista en sala de operaciones, lo que permite marcar endoscópicamente una lesión pequeña que no tiene exteriorización serosa. En el mismo acto operatorio se realizó la colecistectomía por vía laparoscópica, dado el antecedente anteriormente mencionado. Tuvo una buena evolución postoperatoria, con alta a los 5 días.
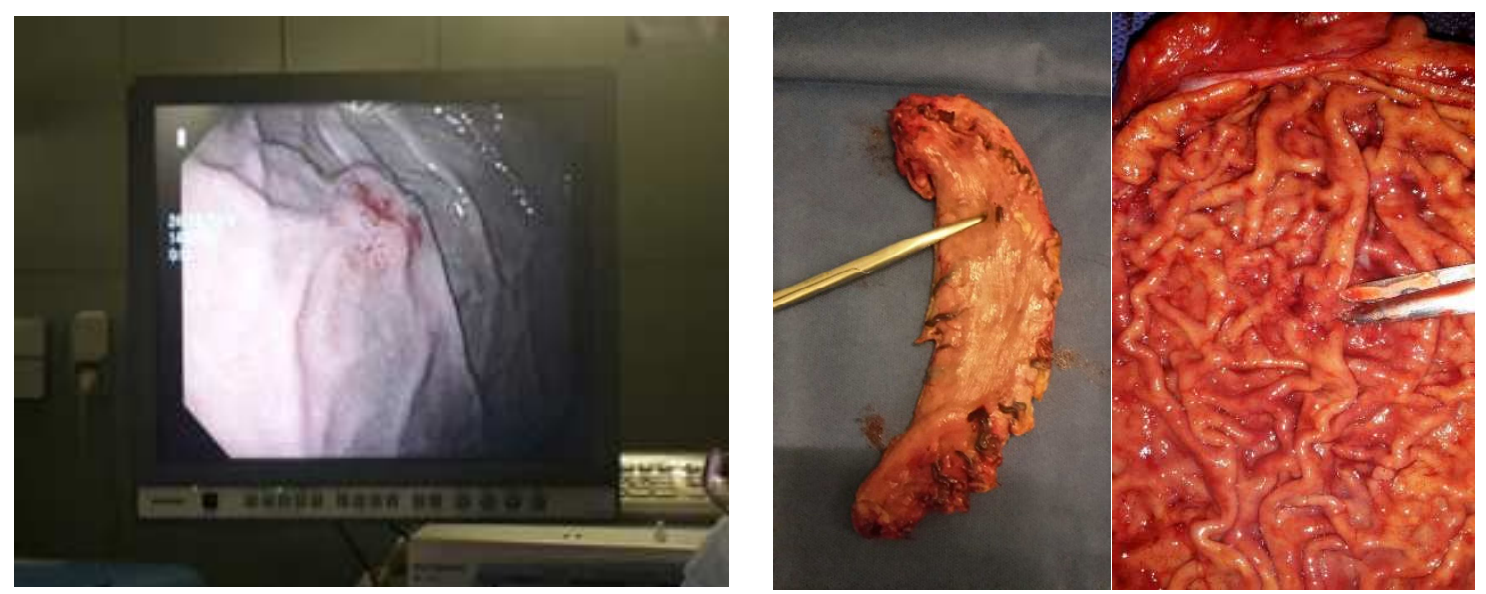

Fig. 4. Endoscopía en sala de operaciones, evidenciando pequeño tumor no palpable sobre curvatura mayor.

Fig. 5. Gastrectomía atípica laparoscópica sobre curvatura mayor.

La AP definitiva muestra un TNE gástrico bien diferenciado grado 1 de la OMS de 11 x 7 mm T2Nx. La IHQ muestra positividad difusa para CromograninaA, sinaptofisina y ckcocktail.

\section{Paciente 3}

Es el caso clínico de una mujer de 63 años con antecedente de gastritis crónica atrófica. Hallazgo en FGC de poliposis gástrica múltiple de distribución difusa. Resección endoscópica de alguno de ellos, cuya anatomía informa TNE gástrico bien diferenciado grado 1 de la OMS (1 mitosis por 10 CGA y Ki67 $2 \%)$.Se completa valoración con PET con Dotatate que no muestra hipercaptación. El dosaje de ácido 5 hidroxi indol acético en orina de 24 hs fue elevado (15 para un normal hasta $9 \mathrm{mg} / 24 \mathrm{hs}$ ).

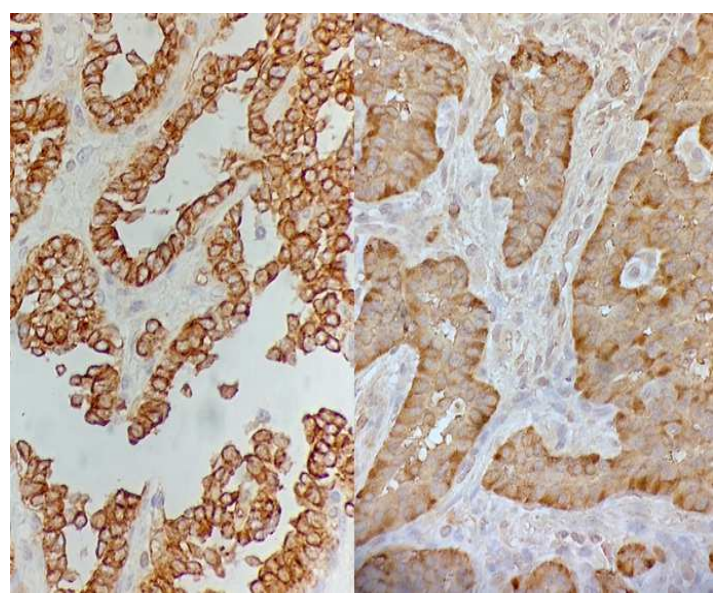

Fig. 6. Pieza: gastrectomía total laparoscópica.

Se decide realizar una cirugía resectiva, gastrectomía total por vía laparoscópica, dada la distribución difusa de los tumores en toda la mucosa gástrica. Cirugía bien tolerada, con anastomosis esófago yeyunal látero lateral con sutura mecánica lineal de tres hileras color violeta. Buena evolución posterior con alta a los siete días con previo tránsito esófago yeyunal al quinto día que no muestra fugas ni estenosis, con buen pasaje distal. 


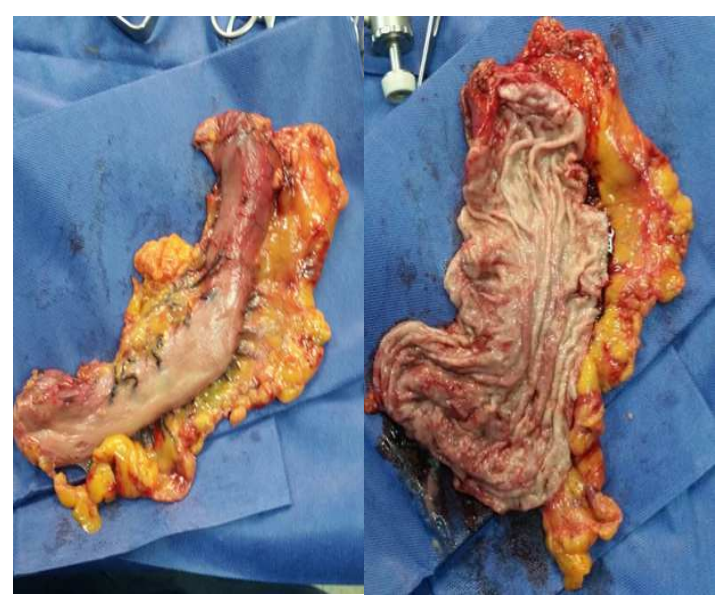

Figura 7. IHQ: ckcocktail + (izquierda), y cromogranina A + (derecha).

La AP definitiva muestra decenas de pequeños pólipos de entre 2-4 mm consistentes con TNE gástrico bien diferenciado múltiple. A la IHQ positividad intensa y difusa para ckcocktail, cromogranina A y sinaptofisina.

\section{Discusión}

Los TNE gástricos suelen ser no secretantes y de pequeño tamaño, por lo que no es raro que sean diagnosticados por un hallazgo imagenológico. En los tres pacientes de nuestra serie, uno de ellos se manifestó por hematemesis, los otros dos fueron hallazgos (uno en una TC abdómino pélvica en el contexto de una apendicitis aguda, y el otro como hallazgo en una FGC en una paciente con antecedente de gastritis erosiva). Está recomendado la dosificación de Gastrina, ya que el aumento de ésta en sangre diferenciará los TNE gástricos tipo 1 y 2 de los tipo 3 (que tienen gastrina normal, y son los de peor pronóstico).

En el estudio de estos tumores la endoscopía digestiva alta es indispensable, permitiendo la mayoría de las vecestopografiar la lesión (si no son submucosos), valorar sus características macroscópicas e incluso en pequeños tumores intentar realizar la resección de los mismos, cuando tienen características endoscópicas favorables (tumores menores a $1 \mathrm{~cm}$, móviles, que no infiltran la capa muscular y sin adenomegalias visibles por otros métodos)(8).La ultrasonografía endoscópica complementa a la endoscopía, valorando la invasión en profundidad y la presencia o no de adenopatías cercanas. Está especialmente indicada cuando los tumores tienen menos de $1 \mathrm{~cm}$ (para valorar el compromiso de la capa muscular ante la eventualidad de resección endoscópica), o ante la localización pancreática o duodenal.

La tomografía computada (TC) con contraste iv debe solicitarse en todos los pacientes. Si bien solo permite topografiar la lesión cuando esta es mayor a $1 \mathrm{~cm}$, situación que no se da la mayoría de las veces (y que se dio en uno de los tres pacientes de la serie), permite descartar adenopatías regionales y la presencia o no de secundarismo hepático (9). Ninguno de los pacientes las tuvo. 
La resonancia magnética nuclear $(\mathrm{RMN})$ tiene mayor sensibilidad para las lesiones pancreáticas y para tipificar lesiones hepáticas subcentimétricas. No fue pedida en nuestros pacientes.

En los tumores desdiferenciados o con un alto índice mitótico, debe solicitarse PET con Dotatate a fin de completar la estadificación. La sensibilidad y especificidad del estudio depende del tipo biológico del tumor. En el caso de la poliposis múltiple se solicitó PET TC, que no mostró hipercaptación. La mayoría de los autores coincide en no solicitar PET TC para los TNE tipo 1 y 2, reservando el estudio para los tumores que muestran diseminación a distancia por tomografía, o los más agresivos (tipo 3) (9). En este caso decidimos solicitar el estudio debido a la gran cantidad de lesiones polipoideas, difíciles de controlar.

Hay cierta discusión sobre la utilidad de la dosificación de Cromogranina A y de ácido 5 hidroxi indol acético (ácido 5HIA) para los TNE gástricos. Algunos autores recomiendan solicitarlo en todos los pacientes, argumentando que apoya el diagnóstico y permite una curva de seguimiento oncológico. El ácido 5HIA solo está elevado en los TNE productores de serotonina, por lo que los valores frecuentemente son normales. Cabe destacar que los valores elevados de Cromogranina A son un factor pronóstico adverso independiente, pero puede dar falso positivo si no se suspenden los inhibidores de la bomba de protones con anterioridad (9).

En tumores gástricos donde el diagnóstico es fundamentalmente histológico, y la biopsia es fácilmente accesible mediante una endoscopía (a diferencia de los TNE pancreáticos o intestinales)puede no ser necesaria la dosificación con criterio diagnóstico de estos marcadores en los TNE grado 1 y 2. Se solicitó Ac 5 HIA en un solo caso, el de los TNE gástricos múltiples, siendo elevado. La mayoría de los autores destaca la utilidad como marcador tumoral del Ac 5HIA en el seguimiento de los tumores agresivos (tipo 3), y como herramienta diagnóstica en los tumores de difícil acceso para la biopsia, en los que un aumento del marcador puede apoyar un diagnóstico imagenológico dudoso.

El tratamiento de elección para los TNE gástricos es la cirugía con resección del tumor. Esta puede ser endoscópica en tumores menores a $1 \mathrm{~cm}$, bien diferenciados, y que no invaden la muscular. La vigilancia endoscópica para este tipo de tumorestambién está aceptada, si hay menos de 6 pólipos vinculados a gastritis crónica atrófica (tipo 1), ya que tienen muy buen pronóstico y casi nulo potencial metastásico(10). Sin embargo, también hay que tener en cuenta la dificultad de un control endoscópico fiable cuando las lesiones son múltiples, por lo que es aceptable la conducta quirúrgica, y debe discutirse con el paciente, el endoscopista y el oncólogo.

En centros con experiencia en cirugía laparoscópica este es un abordaje atractivo, por su menor morbilidad. A diferencia del carcinoma neuroendócrino, los tumores de bajo grado (1 y 2) no tienen indicación de vaciamiento ganglionar, a no ser que se vean adenopatías sospechosas en los estudios paraclínicos o en intraoperatorio.

Los TNE gástricos tienen un pronóstico variable, dado fundamentalmente por su estadio al momento de diagnóstico (TNM) y por su perfil biológico, pero en general mejor que otras localizaciones del tubo digestivo. Los TNE gástricos grado 1 y 2 de la OMS tienen un muy buen pronóstico con sobrevida global a 5 años mayor al $95 \%$ cuando se realiza tratamiento resectivo. Otras localizaciones se asocian a un peor pronóstico (intestino delgado, páncreas, esófago, etc). A diferencia de ellos, solo 
un $4.7 \%$ de los TNE gástricos tienen metástasis al momento de diagnóstico, mientras que los TNE de intestino delgado presentan metástasis hepáticas en un 50-75\% al momento de diagnóstico. (11)

El carcinoma neuroendócrino gástrico (grado 3) tiene muy mal pronóstico, siendo la sobrevida media para los pacientes con enfermedad localizada de 38 meses, y de 5 meses para aquellos que tienen enfermedad a distancia. (12)

Es fundamental contar con patólogos experimentados para el análisis de las biopsias endoscópicas, y de las piezas de anatomía, ya que el pronóstico se establece fundamentalmente por la determinación del grado biológico final de la pieza.

Aún en casos de secundarismo varios trabajos han demostrado un mejor control sintomático y una mejora en la sobrevida global con la resección del tumor primario, por lo que la cirugía está indicada en el caso de ser resecable. (13)Estos pacientes deben ser discutidos en comité de tumores, preferentemente en centros con experiencia, dado que en ocasiones pueden incluso ser sometidos a trasplante hepático con intención curativa (siempre y cuando haya control del primario).

La quimioterapia está indicada en tumores avanzados con diseminación ganglionar o a distancia, generalmente con el uso de platinos.En casos de hipersecreción hormonal el control sintomático mediante análogos de la somatostatina (octeotride) suele ser satisfactorio (14).

En nuestra pequeña serie todos los pacientes tuvieron una buena evolución a largo plazo, con un seguimiento de 48 meses en promedio, sin recidivas.

\section{Conclusiones}

Los TNE gástricos son infrecuentes. Generalmente son bien diferenciados y de bajo potencial maligno. Suelen ser hallazgos en estudios por otras causas, aunque pueden dar síntomas fundamentalmente por sangrado digestivo. El tratamiento de elección es la resección endoscópica o quirúrgica, mediante laparoscopía si el equipo actuante tiene experiencia en su manejo. En caso de tumores bien diferenciados y sin diseminación a distancia el tratamiento quirúrgico es suficiente y no requieren controles oncológicos posteriores. Los tres pacientes presentados en esta comunicación fueron operados por vía laparoscópica, con evolución favorable y sin recidivas en un plazo de 48 meses.

\section{Bibliografía}

1. Siegel R, Miller K, Jemal A. Cancer statistics. CA Cancer J Clin . 2017;67(1):7-30 doi: $10.3322 /$ caac. 21387

2. Sciuto, Pablo y col. Tumores neuroendócrinos del tubo digestivo y páncreas, enfoque quirúrgico. Montevideo: Ediciones de la Plaza, 2015. 
3. Connor JM, Marmissolle F, Bestani C, Pesce V, Belli S, Dominichini E. Observational study of patients with gastroenteropancreatic and bronchial neuroendocrine tumors in Argentina: Results from the large database of a multidisciplinary group clinical multicenter study. Mol Clin Oncol 2014; 2(5):673-84. doi: 10.3892/mco.2014.332

4. Neuroendocrine and Adrenal Tumors. Version 1.2019. In: National Comprehensive Cancer Network (NCCN) Clinical Practice Guidelines in Oncology (NCCN Guidelines). Disponible en: https://arupconsult.com/reference/nccn-neuroendocrine-and-adrenal-tumors-version12019

5. DelleFave G, Kwekkeboom DJ, Van Cutsem E, Rindi G, Kos-Kudla B, Knigge U et al. ENETS Consensus Guidelines for the Management of Patients with Gastroduodenal Neoplasms. Neuroendocrinology 2012; 95:74-87. doi: 10.1159/000335595

6. Young Kim J, Hong SM, Y. Ro J. Recent updates on grading and classification of neuroendocrine tumors. Ann Diagn Pathol 2017;29:11-6. doi: 10.1016/j.anndiagpath.2017.04.005

7. Klöppel, G., Couvelard, A., Perren, A., Komminoth, P., McNicol, A.-M., Nilsson, O, et al.. ENETS Consensus Guidelines for the Standards of Care in Neuroendocrine Tumors: Towards a Standardized Approach to the Diagnosis of Gastroenteropancreatic Neuroendocrine Tumors and Their Prognostic Stratification. Neuroendocrinology, 2009;90(2):162-6. doi: 10.1159/000182196

8. Modlin, I. M., Kidd, M., Latich, I., Zikusoka, M. N., \& Shapiro, M. D. Current Status of Gastrointestinal Carcinoids. Gastroenterology 2005;128(6):171751. doi:10.1053/j.gastro.2005.03.038

9. Kunz PL, Reidy-Lagunes D, Anthony LB, Bertino EM, Brendtro K, Chan JA, et al. Consensus guidelines for the management and treatment of neuroendocrine tumours. Pancreas. 2013;42(4):557-77

10. Modlin, I. M., Oberg, K., Chung, D. C., Jensen, R. T., de Herder, W. W., Thakker, R. V, et bal. Gastroenteropancreatic neuroendocrine tumours. Lancet Oncol 2008; 9(1): 6172. doi:10.1016/s1470-2045(07)70410-2.

11. Modlin IM, Lye KD, Kidd M. A 5-decade analysis of 13715 carcinoid tumors. Cancer 2003; 97: 934-59. doi: 10.1002/cncr.11105.

12. Sorbye, H., Strosberg, J., Baudin, E., Klimstra, D. S., \& Yao, J. C. (2014). Gastroenteropancreatic high-grade neuroendocrine carcinoma. Cancer 2014; 120(18): 2814-23. doi: 10.1002/cncr.28721

13. Madoff DC, Gupta S, Ahrar K, Murthy R, Yao JC. Update on the management of neuroendocrine hepatic metastases. J V Interv Radiol 2006;17(8):1235-49. doi: 10.1097/01.RVI.0000232177.57950.71.

14. Kwekkeboom DJ, de Herder WW, Kam BL, van Eijck CH, van Essen M, Kooij PP, et al. Treatment with the radiolabeled somatostatin analog J Clin Oncol. 2008;26(13):2124-30 doi: 10.1200/JCO.2007.15.2553. 